\title{
Verbal interference in a perceptual comparison task
}

\author{
HOWARD E. EGETH ${ }^{2}$ AND DAVID L. BLECKER, THE JOHNS HOPKINS UNIVERSITY \\ AND ARTHUR S. KAMLET, BEHA VIORAL RESEARCH LABORA TORY, U. S. ARMY HUMAN ENGINEERING LABORATORIES
}

\begin{abstract}
When Ss had to indicate if the colors of pairs of items from the Stroop test were the same or different, the usual interference effect was completely eliminated. However, when the verbal information consisted of SAME and DIFF rather than color names, interference was reestablished. This indicates that the perceptual comparison task does not eliminate interference simply by rendering $S$ s insensitive to the meanings of words.
\end{abstract}

In several recent experiments, the massive interference with color naming that is ordinarily produced in the Stroop (1935) color-word paradigm has been reduced (Pritchatt, 1968) or even completely eliminated (Derks \& Calder, 169) by appropriate manipulation of the S's task. Pritchatt had Ss identify colors with a key-pressing response. When the keys were labeled with the names of the to-be-identified colors, the colors of control rectangles were identified much more quickly than the colors of incongruously colored color words. However, when the keys were labeled with color patches, the difference in response speed between the rectangles and the color words was considerably reduced. Derks and Calder used a counting task in which Ss were asked to indicate the total number of times that a particular color appeared on a sheet. There was essentially no interference produced by the presence of incongruously colored color names.

What is it about these two tasks that makes them relatively impervious to the usual interference produced by the verbal content of incongruous color words? One interesting possibility is that color matching (Pritchatt) and color counting (Derks \& Calder) are "low-level" tasks that do not engage the cognitive apparatus as fully as does the verbal labeling required in the usual Stroop experiment. This line of reasoning leads to the suggestion that Ss simply may not be responsive to the meaning of the words that are present while they are performing in such "low-level" tasks. The present experiment provides a straightforward test of this suggestion and at the same time introduces a new procedure for eliminating the verbal interference produced by incongruously colored color names.

\section{Experiment 1}

\section{METHOD}

Subjects. Ten male BA-level laboratory workers in the Human Engineering Laboratories at Aberdeen Proving Ground volunteered to serve in this experiment.

Stimuli. All stimuli were printed on colored plastic strips (1/2 $\times 1 \frac{1}{4}$ in.) with a Dymo embossing tool. These strips were arranged on white cardboard sheets in five columns of 10 strips each. It should be noted that the embossing process results in white letters appearing on a colored background. The colors (and color words) used were red, yellow, green, and blue.

Conditions. There were six conditions, and for each a 50-item sheet was prepared. In Condition IE, color words were associated randomly with colored backgrounds. (Note: This resulted in about $75 \%$ incongruous associations, whereas most Stroop research has used $100 \%$ incongruity.) In Condition IC, each colored strip had embossed on it four Xs. Thus, in this condition, there was no irrelevant verbal information and it served as a control for the preceding condition. In both of these conditions, Ss were asked to name the colors of the plastic strips as quickly and accurately as possible. In Condition IIE, the 50 strips were arranged in 25 pairs, one member of a pair directly above the other, and Ss were asked to state for each pair whether the colors of the strips were the same or different. The colors and words for this condition were selected to ensure that: (1) for about half of the pairs the correct response was "same," and (2) for about half of all pairs the verbal information was inconsistent with the color information, while for the other half it was consistent. As an example of inconsistent information, consider a pair in which both members are blue, but the top member reads blue while the bottom member reads green. The correct answer is "same" because the colors are the same, however, the two words are different and to the extent that a $S$ comprehends their meaning he may be led to respond "different." Condition IIC was designed to serve as a control for the preceding condition; the colored strips were arranged similarly, but they contained Xs rather than color words.

In Condition IIIE, color patches were again arranged in pairs and Ss were required to judge whether the colors were same or different, but instead of color words, the word SAME and the abbreviation DIFF were used. As in Conditions IIE and IIC, the pairs were designed so that for about half of them the correct response was "same" and for the other half "different." The verbal material was introduced in such a way that it was inconsistent with the color information on about half of the trials and consistent on the other half. As an example of inconsistent information, consider a pair in which both members are red but both say DIFF. The correct answer is "same" because the colors are the same, but the verbal material may obtrude itself on the $S$ and prompt him to respond "different." Unlike the situation in Conditions IIE and IIC, it seemed appropriate here to use the identical word for both members of a given pair. Condition IIIC, which was actually the same as Condition IIC, served as the control for Condition IIIE.

Procedure. The Ss were given the tasks in the order IC, IE, IIC, IIE, IIIC, IIIE, with a 1.5-min break between trials. Following a 2.5-min rest, they were then run through the tasks again in a similar fashion and in the same order. Their instructions stressed both speed and accuracy, and they were told to correct any errors as they went along. Timing was done with a stopwatch.

\section{Experiment 2}

Essentially the same experiment was performed with 12 Johns Hopkins undergraduates serving as Ss. There were, however, several procedural differences. For one, the lettering was done with a silk-screen process and thus the words themselves, rather than their backgrounds, were colored. The words were considerably larger and there were only 48 items (or 24 pairs) to a sheet. The order of presentation of conditions was random with the restriction that a task (E) and its control (C) were always adjacent. The Ss were again run through all of the conditions twice, in two randomized blocks. Finally, the Es differed in the two experiments.

\section{RESULTS AND DISCUSSION}

No particularly interesting practice effects were evident, and so the data from the two blocks were combined. In Table 1, the mean response time for each of the six conditions is shown separately for the two experiments. In examining these data it 
Table 1

Response Times for All Conditions and Percentage Decrements for Experimental Conditions. Percentage Decrement is Difference Between Experimental and Control Response Times Divided by Control Response Time

\begin{tabular}{llcccc} 
& & & & \multicolumn{2}{c}{$\begin{array}{c}\text { Percentage } \\
\text { Decrement }\end{array}$} \\
\cline { 3 - 6 } & & Exp. I & Exp. II & Exp. I & Exp. II \\
IC & Stroop (Control) & 23.9 & 25.4 & & \\
IE & Stroop & 37.5 & 37.9 & $57^{*}$ & $49^{*}$ \\
IIC & Comparison (Control) & 14.5 & 15.2 & & \\
IIE & Comparison (Color Words) & 13.8 & 15.3 & $-5 \mathrm{~ns}$ & $1 \mathrm{~ns}$ \\
IIIC & Comparison (Control) & 13.8 & 14.7 & & \\
IIIE & Comparison (SAME DIFF) & 17.7 & 17.8 & $28^{*}$ & $21^{*}$ \\
\hline
\end{tabular}

$* p<01$

ns $p<.10$

should be remembered that the comparative tasks (II and III) require only half the number of overt responses of the ordinary color-identification task (I) since the items are arranged in pairs. In addition to the mean times, the data for each experimental condition are expressed in terms of percentage decrement with respect to its corresponding control condition.

In addition to the obvious reconfirmation of the Stroop effect (IE vs IC), there are two important pieces of information in these data. First, incongruous color names seem to cause no interference at all with performance in the color-comparison task used in this experiment (IIE vs IIC). On these grounds, the task seems to provide an excellent testing ground for the hypothesis mentioned in the introduction. Second, there is a substantial degree of interference in the comparison task when the verbal information is provided by the words SAME and DIFF (IIIE vs IIIC). These statements are verified both by the closeness of replication between Experiments 1 and 2, and by tests for matched samples (where significance is implied, $\mathrm{p}<.01$; where nonsignificance is implied, $\mathrm{p}>.10$ ).

Since the words SAME and DIFF do cause interference, it is clear that the Ss were not insensitive to verbal meaning per se in the perceptual comparison task used in this experiment. However, these data leave another important issue unresolved. This is the question of whether the perception of particular words is dependent upon their closeness in meaning to the words that are used as responses in the task. It may be the case that all of the words used in the present study were equally well perceived and that degree of interference is a function of processes subsequent to word recognition. However, it is also conceivable that word recognition is itself dependent on the semantic similarity of the spoken and printed words. Further research is necessary to evaluate these alternative descriptions.

\section{Further Comment}

The complete lack of interference observed in Condition IIE deserves further discussion. One might think it due simply to the color words being effectively unrelated to the response words "same" and "different." However, as Klein (1964) has shown, in the usual Stroop color-naming task the presence of familiar words is distracting, even when these words are not directly related to the response words. This discrepancy between the present data and Klein's suggests that the nature of the S's task is, in fact, a determinant of the degree to which irrelevant information is interfering.

The counting task used by Derks and Calder provides another example of a task that seems to be relatively impervious to interference. The present authors have tried the color-counting task with sheets of material like those used in Experiment 1, but with the verbal material consisting of numerals. The data failed to indicate any interference due to the presence of the potentially interfering numerals. Why did the numerals fail to produce interference while SAME and DIFF did produce interference in Condition IIIE of the main experiment? The reason is probably that the counting task, unlike labeling or rendering a comparative judgment, can be accomplished so quickly that the meaning of the verbal material simply does not have time to produce interference.

\section{REFERENCES}

DERKS, P. L., \& CALDER, E. S. Information processing and verbal labels: The Stroop color-word test. Paper presented at the meeting of the Eastern Psychological Association, Philadelphia, 1969.

KLEIN, G. S. Semantic power measured through the interference of words with color-naming. American Journal of Psychology, 1964, 77, 576-588.

PRITCHATT, D. An investigation in to some of the underlying associative processes of the Stroop Colour Effect. Quarterly Journal of Experimental Psychology, 1968, 20, 351-359.

STROOP, J. R. Studies of interference in serial verbal reactions. Journal of Experimental Psychology, 1935, 18, 643-662.

\section{NOTES}

1. This research was supported in part by grants to the senior author from the National Science Foundation and the Office of Education. A version of this paper was presented at the meeting of the Eastern Psychological Association, Philadelphia, 1969.

2. Address: Department of Psychology, The Johns Hopkins University, Baltimore, Maryland 21218.

(Accepted for publication A pril 28, 1969.) 